HNO 2013 $61: 592-592$

DOI 10.1007/s00106-013-2690-7

Online publiziert: 14. März 2013

(c) Springer-Verlag Berlin Heidelberg 2013

J. Löhler ${ }^{1}$ B. Akcicek ${ }^{1} \cdot$ M. Pilnik ${ }^{1} \cdot$ K. Saager-Post ${ }^{1} \cdot$ S. Dazert ${ }^{2} \cdot$ S. Biedron ${ }^{2} \cdot$

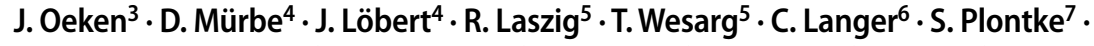

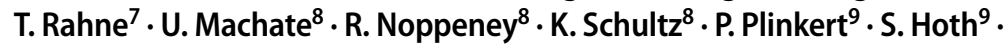

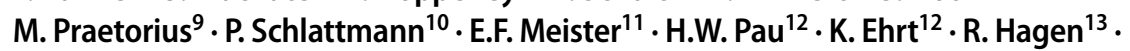
W. Shehata-Dieler ${ }^{13} \cdot$ M. Cebulla ${ }^{13} \cdot$ L.E. Walther ${ }^{14} \cdot$ A. Ernst ${ }^{15}$

${ }^{1}$ HNO-Praxis, Wissenschaftliches Institut für angewandte HNO-Heilkunde (WIAHNO), Bad Bramstedt

${ }^{2}$ St.-Elisabeth-Hospital, HNO-Universitätsklinikum Bochum

${ }^{3}$ HNO-Klinikum Chemnitz

${ }^{4}$ HNO-Universitätsklinikum Dresden

${ }^{5}$ HNO-Universitätsklinikum Freiburg

${ }^{6}$ HNO-Praxis, Füssen

${ }^{7}$ HNO-Universitätsklinikum Halle (Saale)

${ }^{8}$ HNO-Gemeinschaftspraxis, Hamburg-Mundsburg

${ }^{9}$ HNO-Universitätsklinikum Heidelberg

${ }^{10}$ Institut für Medizinische Statistik, Informatik und Dokumentation, Universitätsklinik Jena

${ }^{11}$ HNO-Klinik, Klinikum St. Georg, Leipzig

${ }^{12}$ HNO-Universitätsklinikum Rostock

${ }^{13}$ HNO-Universitätsklinikum Würzburg

${ }^{14}$ HNO-Zentrum, Sulzbach/Taunus

${ }^{15}$ HNO-Klinik, Unfallkrankenhaus Berlin

\title{
Erratum zu: Evaluation des Freiburger Einsilbertests im Störschall
}

\author{
HNO Online First, 19. Oktober 2012 \\ http://dx.doi.org/10.1007/s00106-012-2598-7
}

In der HTML-Version des o. g. Beitrags sind leider unvollständige Angaben gemacht worden bzw. folgende Ergänzungen erforderlich:

- Bei den Autoren muss Meister EF als Autor nach Schlattmann P ergänzt werden, die Autorenreihung entspricht also denen des hier abgedruckten Erratums.

- Der Begriff „Heil- und Hilfsmittelrichtlinien“ muss im gesamten Text durch „Hilfsmittelrichtlinien“ ersetzt werden.

- Im Teil Methoden fehlt im zweiten Satz das Wort „wurden“.

- Im englischen Abstract unter Results muss es heißen „p $<0,05$ “ statt „p $>0,05$ “.
In der Printversion wurden diese Änderungen bereits berücksichtigt. Wir bitten darum, die Fehler zu entschuldigen.

\section{Korrespondenzadresse}

\section{Dr.J. Löhler}

HNO-Praxis, Wissenschaftliches Institut für angewandte HNO-Heilkunde (WIAHNO) Maienbeeck 1, 24576 Bad Bramstedt jan.loehler@hnoarzt-bad-bramstedt.de 